\title{
Widely tunable optical properties via oxygen manipulation in an amorphous alloy
}

\author{
Ying-qi Zhang ${ }^{1}$, Li-ying Zhou ${ }^{2,3}$, Sheng-ye Tao ${ }^{1}$, Yu-zhang Jiao ${ }^{1}$, Jin-feng Li ${ }^{4}$, Kai-ming Zheng ${ }^{1}$, \\ Yuan-chao Hu ${ }^{5}$, Kai-xuan Fang ${ }^{1}$, Cheng Song ${ }^{6}$, Xiao-yan Zhong ${ }^{7,8}$, Limei Xu ${ }^{2,3^{*}}$, Ke-Fu Yao ${ }^{1}$, \\ Zheng-jun Zhang ${ }^{6}$ and $\mathrm{Na} \mathrm{Chen}^{{ }^{*}}$
}

\begin{abstract}
The ability to widely tune the optical properties of amorphous alloys is highly desirable especially for their potential applications in optoelectronic devices. In this work, we demonstrate that introducing oxygen into an amorphous alloy system of Co-Fe-Ta-B enables the formation of various amorphous derivatives ranging from metals to semiconductors, and eventually to insulators. These oxygencontaining amorphous derivatives gradually become transparent with the opened bandgaps, leading to a continuous increase in their optical transmittance. Furthermore, the reflective metal-type amorphous alloy and transparent insulator-type amorphous oxide of the system can be integrated together to realize the full-color tuning over the entire visible spectral range. This provides a new way to develop large-area color coatings with high design flexibility and full-color tunability. We envisage that the design concept proposed in this work is also applicable to many other amorphous alloy systems, from which all types of amorphous materials including alloys, semiconductors and insulators may be developed to show unprecedented optical functionalities.
\end{abstract}

Keywords: amorphous alloy, oxygen manipulation, amorphous oxide, optical properties

\section{INTRODUCTION}

Unlike crystals with periodic lattice structures, amorphous thin films possess long-range disordered structures
[1-4]. They are very useful in various fields including optics, electronics and catalysis due to their fruitful kinds ranging from metals to insulators [5-11]. Nevertheless, it is difficult to widely tune their properties via the amorphous structural manipulation [12-16].

Previous studies reported that a ferromagnetic amorphous alloy (AA) was converted into a single-phase amorphous oxide (AO) ferromagnetic semiconductor with a bandgap of $\sim 2.4 \mathrm{eV}$ through severe oxidization $[17,18]$. It was because the AA was mainly characterized by the metallic bonds. Such non-directional and nonsaturated atomic bonding could allow a large amount of oxygen to be continuously added into the AA. As a result, the developed AOs may become optically transparent wide-bandgap semiconductors at sufficiently high oxygen contents. In fact, transparent AOs are thought to meet the demands for developing transparent and flexible optoelectronic devices such as rolled-up displays and solar cells [19-23]. Therefore, developing transparent AOs from AAs may offer an opportunity to generate combinational functionalities including high hardness and high strength inherited from the AA hosts, which are useful for enhancing the service reliability of the optoelectronic devices.

In the present work, we showed that the structures and optical properties of the AA Co-Fe-Ta-B system were widely tuned via oxygen manipulation in a controlled

\footnotetext{
${ }^{1}$ Key Laboratory for Advanced Materials Processing Technology (MOE), The State Key Laboratory of New Ceramics and Fine Processing, School of Materials Science and Engineering, Tsinghua University, Beijing 100084, China

${ }^{2}$ International Center for Quantum Materials, Peking University, Beijing 100084, China

${ }^{3}$ Collaborative Innovation Center of Quantum Matter, Beijing 100871, China

${ }^{4}$ Institute of Materials, China Academy of Engineering Physics, Mianyang 621907, China

${ }^{5}$ Institute of Industrial Science, University of Tokyo, 4-6-1 Komaba, Meguro-ku, Tokyo 1538505, Japan

${ }^{6}$ Key Laboratory for Advanced Materials (MOE), School of Materials Science and Engineering, Tsinghua University, Beijing 100084, China

${ }^{7}$ National Center for Electron Microscopy in Beijing, Key Laboratory of Advanced Materials (MOE), The State Key Laboratory of New Ceramics and Fine Processing, School of Materials Science and Engineering, Tsinghua University, Beijing 100084, China

${ }^{8}$ Department of Materials Science and Engineering, City University of Hong Kong, Tat Chee Avenue, Kowloon, Hong Kong, China

* Corresponding authors (emails: limei.xu@pku.edu.cn (Xu L); chennadm@mail.tsinghua.edu.cn (Chen N))
} 
manner. At high oxygen content levels over 50.6 at.\%, transparent AOs were developed from the AA host. Furthermore, the reflective AA and transparent $\mathrm{AO}$ of the system were combined to form bilayer color coatings with the full-color tunability.

\section{EXPERIMENTAL SECTION}

The Co-Fe-Ta-B-O ${ }_{x}\left(\mathrm{CFTBO}_{x}\right)$ thin films were fabricated at room temperature by magnetron sputter deposition from an alloy target with a nominal composition of $\mathrm{Co}_{45} \mathrm{Fe}_{21} \mathrm{Ta}_{10} \mathrm{~B}_{24}$ (at.\%) in a gas mixture of argon and oxygen. The base pressure before deposition was $\sim 3 \times 10^{-5} \mathrm{~Pa}$. The oxygen content level of the as-deposited thin film was adjusted by varying the oxygen partial pressure in the working gas mixture. The substrate was rotated during the sputtering to ensure the compositional uniformity.

The thickness of the thin films was measured by an atomic force microscope (AFM, Cypher ES, OXFORD INSTRUMENTS of UK). The compositions of the asdeposited thin films were examined by an auger electron spectroscopy (AES, PHI 710, ULVAC-PHI of Japan). The structures of the thin films were investigated by using $\mathrm{X}$ ray diffractometer (XRD, D/max $2500 \mathrm{H}$, RIGAKU CORPORATION of Japan) and high-resolution transmission electron microscopy (HRTEM, JEM-2100, JEOL of Japan). The optical properties were measured by using a UV/VIS spectrophotometer (Lambda 950, PerkinElmer of USA) and an ellipsometer (VASE, J. A. Woollam of USA), respectively.

\section{RESULTS AND DISCUSSION}

Based on the alloy system of Co-Fe-Ta-B (CFTB), the compositions and corresponding structures of the developed $(\mathrm{CFTB})_{100-x} \mathrm{O}_{x}$ (abbreviated as $\mathrm{CFTBO}_{x}, 0<x \leq$ $60.0)$ thin films were tuned in a well-controlled manner. Note that the highest oxygen content accessed in the present study was 60.0 at.\%, likely due to the technical limitation or the existence of the upper limit for the oxygen content to be involved in the system. Fig. 1a shows the XRD patterns of the $\mathrm{CFTBO}_{x}$ thin films. As $x$ increases, the structures of these thin films evolved from a
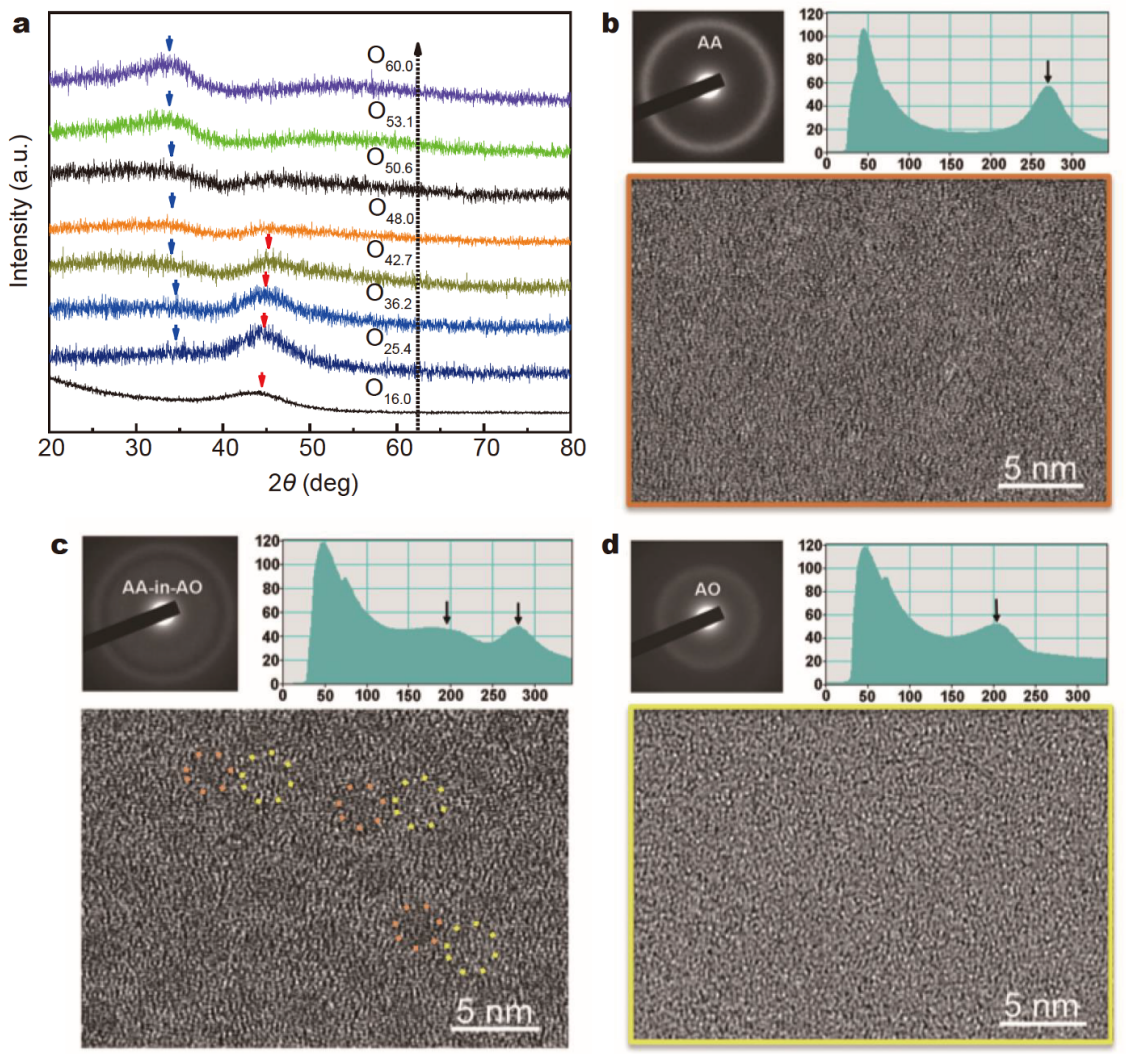

Figure 1 Structural characterization of the $\mathrm{CFTBO}_{x}$ thin films. (a) XRD patterns of the thin films with different oxygen contents. (b-d) HRTEM images taken for the thin films at the oxygen contents of 16.0 at.\%, 36.2 at.\% and 48.0 at.\%. The SAED patterns and their digitized intensity profiles are shown in the upper parts of (b-d), respectively. 
single-phase AA to dual-phase structures consisting of the $\mathrm{AA}$ and $\mathrm{AO}$, and eventually to a single-phase $\mathrm{AO}$ (Fig. 1a). With $x$ no larger than 16.0 , the $\mathrm{CFTBO}_{x}$ possessed a single-phase AA structure as further confirmed by its HRTEM image (Fig. 1b). Increasing $x$ to above 16.0 enabled the formation of an $\mathrm{AO}$ phase, whose volume fraction increased with $x$. As a result, supra-nanometersized dual-phase composites were formed, exhibiting the structures of ultrafine AA nano-granules embedded in the AO matrix (AA-in-AO, Fig. 1c). Eventually, the AA phase transferred into single-phase AOs with $x \geq 48.0$ (Fig. 1d). The upper parts of Fig. $1 \mathrm{~b}-\mathrm{d}$ are the selected area electron diffraction (SAED) patterns and their digitized intensity profiles, which confirmed the structural evolution from the single AA phase to the dual-phase nanocomposite, and to the single $\mathrm{AO}$ phase in these $\mathrm{CFTBO}_{x}$ thin films.

The structural characterization of these $\mathrm{CFTBO}_{x}$ thin films indicated that increasing $x$ enabled the $\mathrm{CFTBO}_{x}$ system to be gradually oxidized. Hence, the continuous oxidization localized more and more freely movable charge carriers, leading to a gradual decrease in the number of the charge carriers per mole $\left(n_{\mathrm{tc}}\right)$. This altered the amorphous system's electronic band structures from the metal-type for the AAs to the insulator-type for the
AOs. Therefore, the optical bandgap $\left(E_{\mathrm{g}}\right)$ of this system would be opened with the changes in $n_{\mathrm{tc}}$ as shown below.

$$
\begin{aligned}
n_{\mathrm{tc}} & =\left(1-\frac{x}{100}\right) n_{\mathrm{tc}}^{0}-a N_{\mathrm{A}} \frac{x}{100} \\
& =n_{\mathrm{tc}}^{0}-\left(n_{\mathrm{tc}}^{0}+a N_{\mathrm{A}}\right) \frac{x}{100},
\end{aligned}
$$

$E_{\mathrm{g}} \propto \frac{1}{n_{\mathrm{tc}}} \propto x$

where $n_{\text {tc }}^{0}$ denotes the number of charge carriers per mole for the AA host without oxygen, $a$ denotes the average valence electron number localized by an oxygen atom, $N_{\mathrm{A}}$ denotes the Avogadro constant. According to the above equations, the optical transparency of the system should increase with $x$ because of the opened optical bandgap.

As shown in Fig. 2a, the $\mathrm{CFTBO}_{x}$ thin films indeed became more and more transparent with increasing $x$ up to 60.0. Their appearance varied from opaque metal luster to high transparency. Correspondingly, the transmittance in the visible light range for the as-prepared thin films increased with $x(0<x \leq 60.0)$ as shown in Fig. 2b. The normal sighted human eye is most sensitive to the visible light at the wavelength of $555 \mathrm{~nm}$. Based on Fig. 2b, the derived optical transmittance at $555 \mathrm{~nm}$ for the $\mathrm{CFTBO}_{x}$
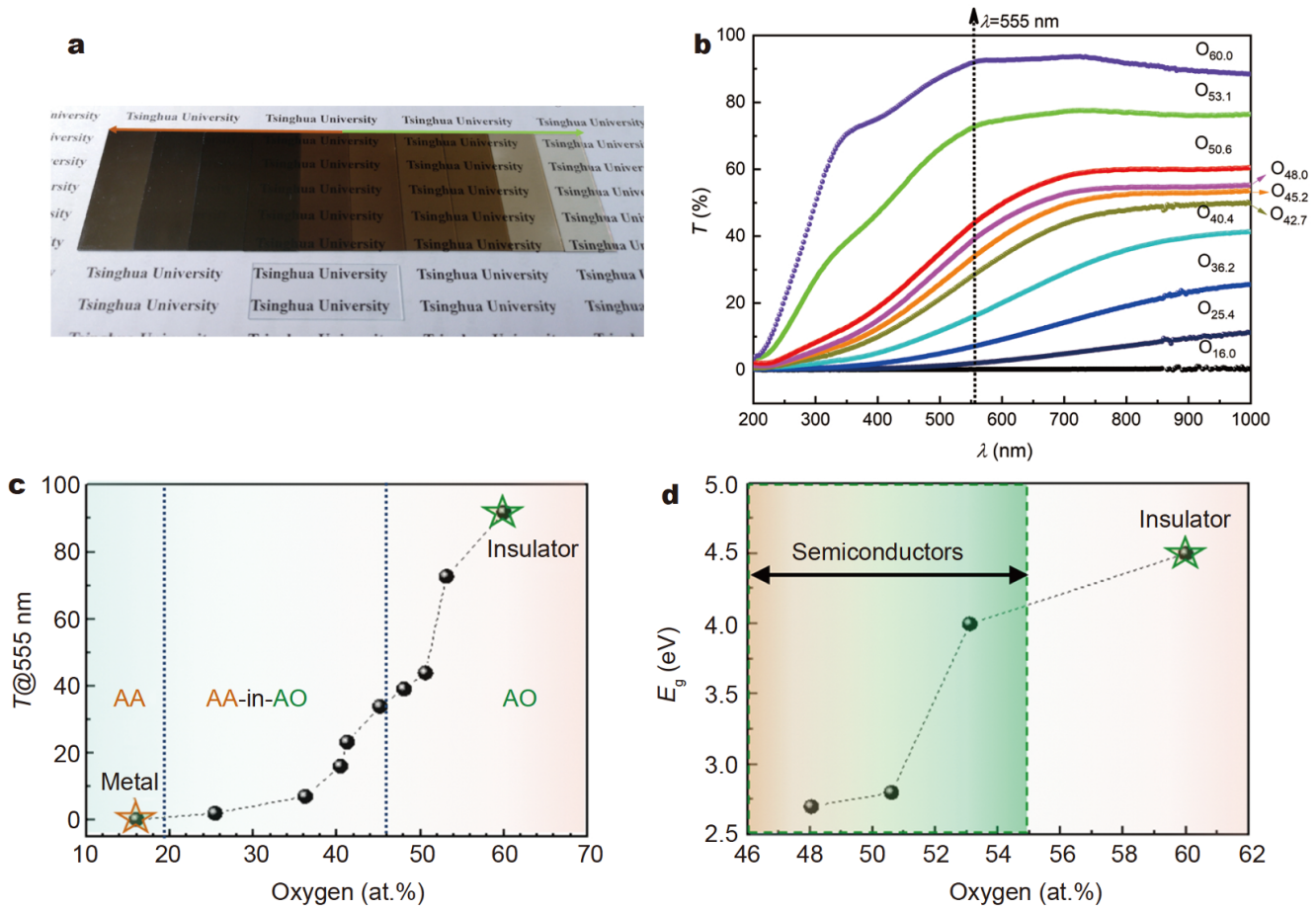

Figure 2 Optical properties of the $\mathrm{CFTBO}_{x}$ thin films. (a) The appearance of the thin films at different oxygen contents. (b) The optical transmittance $(T)$ varying with the wavelength in the visible light range. (c) The optical transmittance at the wavelength of $550 \mathrm{~nm}$ and (d) the optical bandgap varying with different oxygen contents. 
thin films increased with $x(0<x \leq 60.0)$ as well (Fig. 2c). The $\mathrm{CFTBO}_{60.0}$ thin film showed the transmittance of about $90 \%$, the highest value among the as-deposited thin films with the oxygen contents no more than 60.0 at.\%. As predicted by Equation (2), the $E_{\mathrm{g}}$ for the single-phase AO thin films with $x \geq 48.0$ increased from $\sim 2.7$ to $\sim 4.5 \mathrm{eV}$ with $x$ (Fig. $2 \mathrm{~d}$ ).

Utilizing the significantly different optical properties of the metallic AA $\left(\mathrm{CFTBO}_{16.0}\right)$ and insulating $\mathrm{AO}$ $\left(\mathrm{CFTBO}_{53.1}\right)$ thin films, full-color coatings were formed. The insulating AO thin film showed high optical trans- mittance within the visible light range and thus functioned as a dielectric medium for the light to transmit (Fig. 2a, b). The AA thin film showed high optical reflectivity in the visible light range and thus behaved as a light reflector (see Fig. 3a). A schematic diagram for this bilayer structure of the AA and AO thin films is shown in the inset of Fig. 3a. The incidence light was reflected and refracted at the surface of the AO thin film, respectively. The refracted light wave entered the AO thin film and was then reflected from the AA surface. The reflected light was refracted once more at the surface of the $\mathrm{AO}$
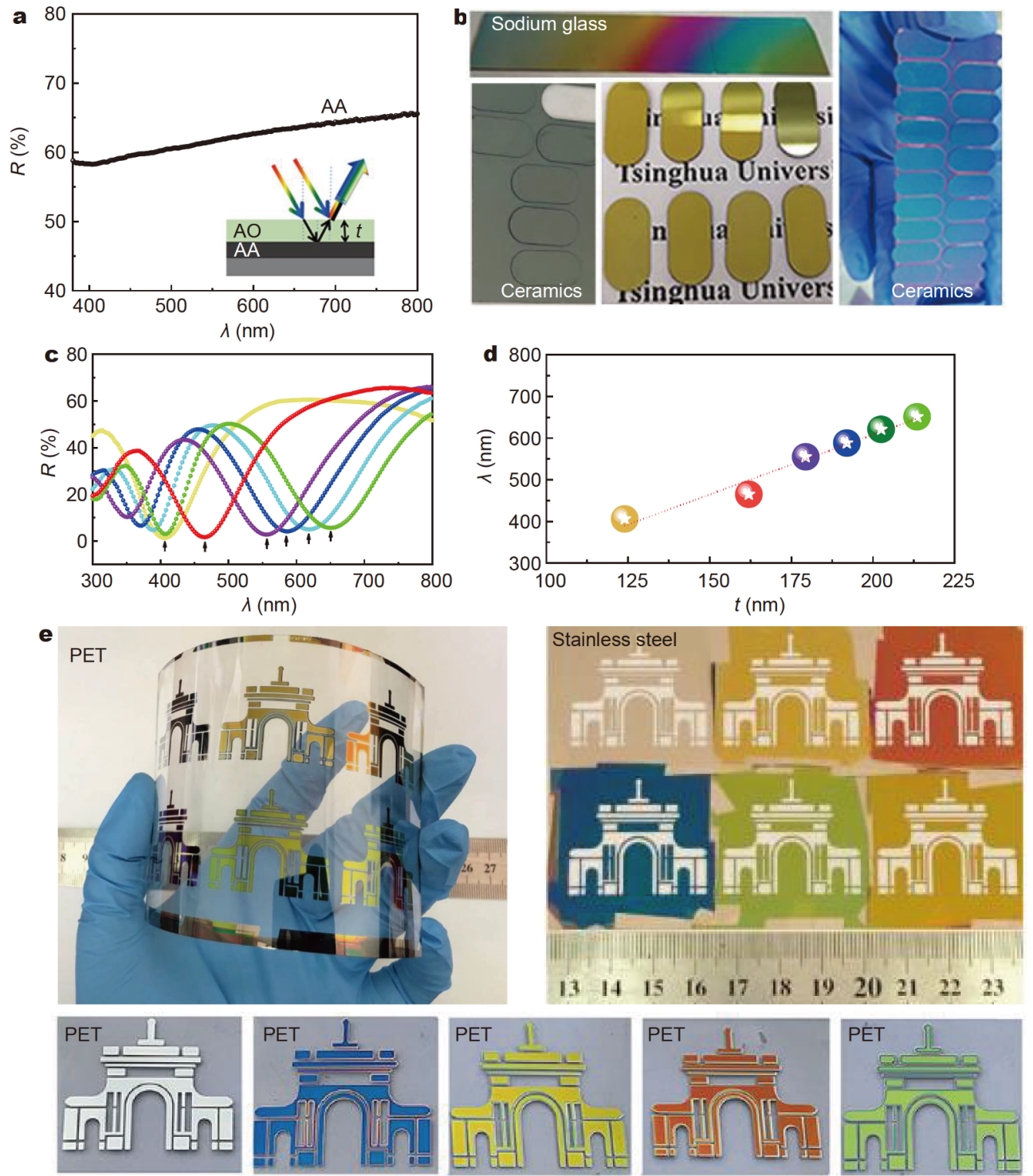

Figure 3 (a) The resistivity spectrum measured for the AA thin film. Inset is a schematic diagram for a bilayer structure consisting of the AA and AO thin films. (b) Rainbow colors observed on the sodium glass and various colors observed on the $\mathrm{Al}_{2} \mathrm{O}_{3}$ ceramic plates, respectively. (c) The optical reflectivity spectra measured for the different color coatings. (d) The wavelength $(\lambda)$ of the resistivity minimum almost linearly correlated with the thickness $(t)$. (e) Various colors observed on the stainless steel and flexible PET film substrates. 
thin film as shown in the inset of Fig. 3a. The refracted light encountered the reflected light at the surface of the AO layer, resulting in the thin film interference effect.

Such effect often results in colorful patterns on soap bubbles or oil films in our daily life. The presence of specific colors is mainly determined by the optical path difference between the reflected and refracted light waves, which is closely related to the thickness $(t)$ of the AO thin film. Therefore, adjusting $t$ enabled the observation of different colors on sodium glass and $\mathrm{Al}_{2} \mathrm{O}_{3}$ ceramic plates as shown in Fig. 3b. Rainbow-like colors appeared on the sodium glass by depositing the bilayer thin films consisting of the AO thin film with continuously varied $t$. In addition, various colors including dark-grey, yellow and blue were obtained by depositing the bilayer thin films comprising the $\mathrm{AO}$ thin films with determined $t$ on the ceramics, respectively. Based on the reflectivity spectra measured for different colors shown in Fig. 3c, the wavelength $(\lambda)$ at the reflectivity minimum can be linearly correlated with $t$ (Fig. 3d). Accordingly, depositing the bilayer thin films with the $\mathrm{AO}$ thin films at the predetermined $t$ enabled the flexible polyester (PET) and stainless steel substrates to display various colors such as white, red, blue, orange and green with high resolution and brightness, respectively (Fig. 3e).

Considering its electronically insulating characteristic, we also deposited the $\mathrm{AO}$ thin films on intrinsic and n-type/p-type Si substrates, respectively. Similar to the above bilayer structures, the $\mathrm{Si}$ substrates behaved as a reflector while the AO thin films functioned as an optically transmitting layer. Varying $t$ of the top AO thin film affected the optical path difference and thus enabled the observation of various colors of blue, white, yellow, red, purple and green (Fig. 4a, b). Meanwhile, the colorful p-n and $\mathrm{p}-\mathrm{p}$ heterojunctions exhibited their corresponding electrical behaviors such as on/off switching and resistance functionalities, respectively (Fig. $4 \mathrm{c}-\mathrm{e}$ ). Si is one of the key semiconductor materials in thin-film semiconductor devices. The formation of these colorful $\mathrm{p}-\mathrm{n} / \mathrm{p}-\mathrm{p}$ heterojunctions shows their possibility to be integrated in thin-film optoelectronic devices. Especially, the colorful p-n heterojunctions combine both switching and color filtering functionalities. As the switches, they can control the electrical current to flow or not to realize the signal processing. Meanwhile, they can exhibit various colors by tuning the thickness of the AO thin film. In this way, using only one component based on these colorful $\mathrm{p}-\mathrm{n}$ heterojunctions may partially replace the two components of the data processing and pixel units currently used in the flat-panel displays.

Furthermore, the optical reflectivity $(R)$ spectra for the above color coatings on the $\mathrm{Si}$ substrates were measured as shown in Fig. 5a, exhibiting different reflectivity minimum and maximum in the visible light range, respectively. The wavelength $(\lambda)$ at the reflectivity minimum was determined for different colors as indicated by the black arrows in Fig. 5a. Similar to the bilayer structures of the AA and $\mathrm{AO}$ thin films, $t$ of the AO thin film
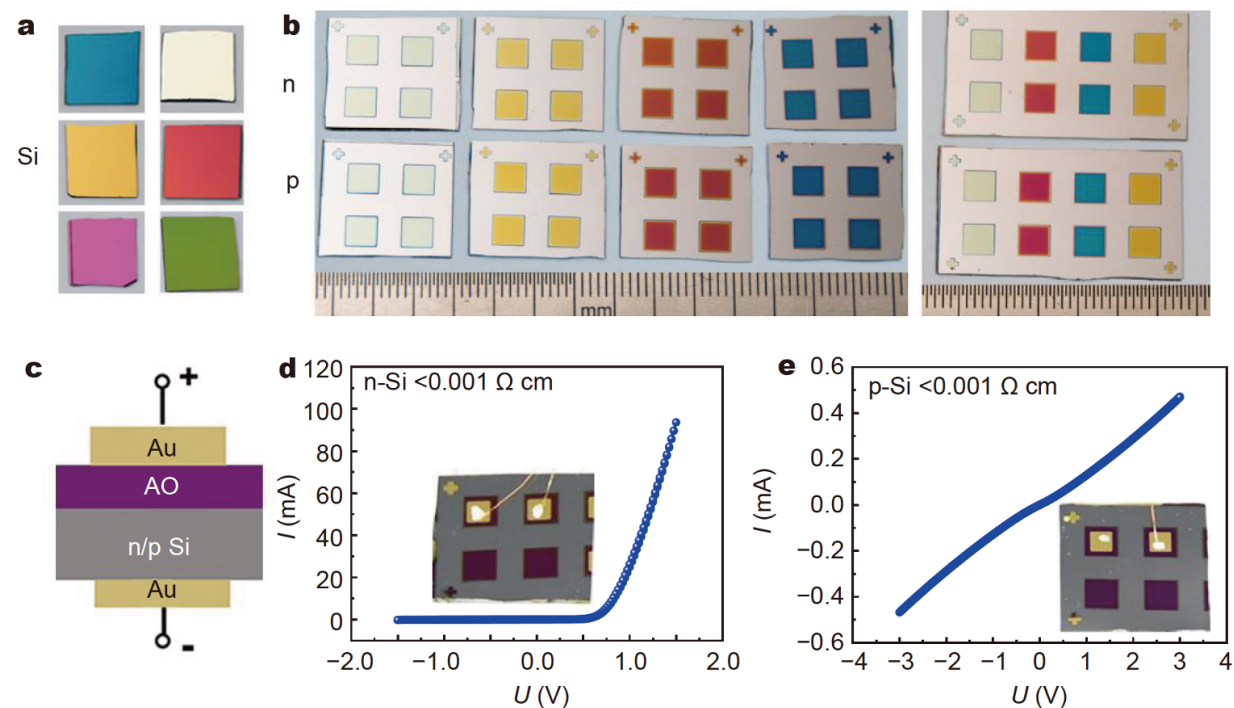

Figure 4 (a, b) Various color coatings formed by depositing the AO thin films on intrinsic Si and p-type/n-type Si substrates, respectively. (c) Schematic diagram for measuring $I-V$ curves of the p-n/p-p heterojunctions. (d, e) The $I-V$ curves of the heterojunctions showing the rectification and resistance behaviors, respectively. 

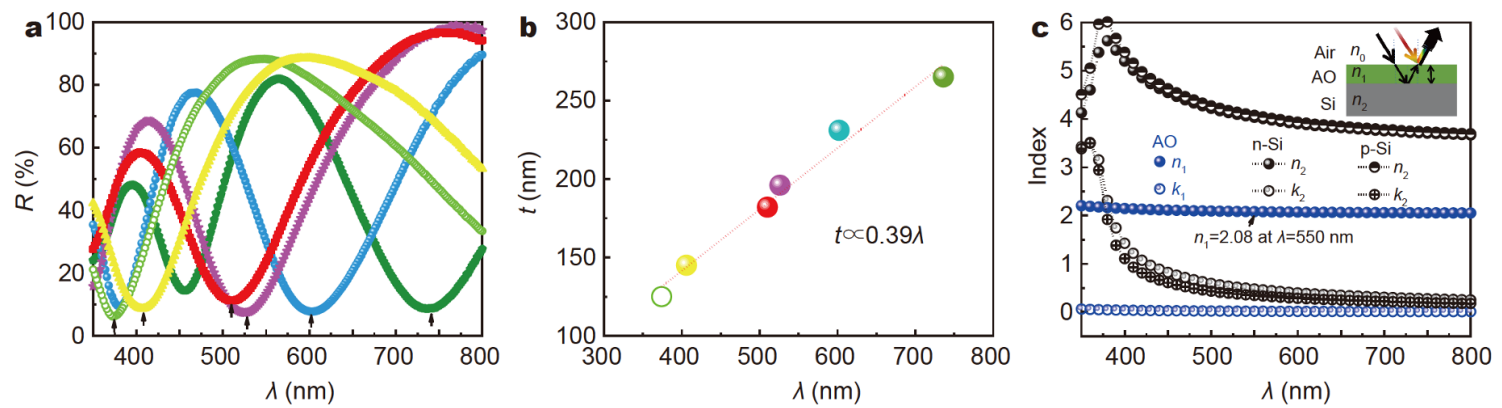

Figure 5 (a) The reflectivity spectra for different color coatings. (b) The correlation between the thickness $(t)$ and the wavelength $(\lambda)$ of the reflectivity minimum. (c) The refractive indices $(n)$ and extinction coefficients $(k)$ measured for the AO thin film and the p-type/n-type Si substrates, respectively.

can be linearly correlated with $\lambda$ as well (Fig. 5b). The slope of the fitted line is about 0.39 .

As shown in Fig. $5 c$, the refractive indices $(n)$ and extinction coefficients $(k)$ of the AO thin film, n-type Si and p-type Si were measured, respectively. Note that both the n-type and p-type Si substrates showed almost the same $n$ and $k$ values within the visible-light range. The $\mathrm{AO}$ thin film showed nearly zero- $k$, indicating that there was almost no loss for the visible light waves to transmit through it. Meanwhile, its $n$ values were almost unchanged and were smaller than those of the Si substrates. When an incident light encountered the interface from the optically thinner medium (air, $n_{0}=1$ ) onto the optically denser AO thin film $\left(n_{1}=2.08\right.$ at $\left.\lambda=550 \mathrm{~nm}\right)$, the reflected light wave would have a phase shift of $\pi$, corresponding to a half-wave loss [24]. Similarly, the light wave would experience the second half-wave loss at the interface between the AO thin film $\left(n_{1}=2.08\right.$ at $\lambda$ $=550 \mathrm{~nm})$ and the Si substrate $\left(n_{2}=4.04\right.$ at $\left.\lambda=550 \mathrm{~nm}\right)$. In this case, a destructive interference effect would occur for the AO thin films with quarter-wave thickness $(t)$ in terms of $t \cong \frac{(2 m+1)}{4 n_{1}} \lambda(m=0,1,2,3 \ldots)$ [25], which resulted in the reflectivity minimum at $\lambda$. Hence, the AO thin film would present the complementary color of the visible light wave with $\lambda$. Taking into account almost unchanged $n_{1}$ within the visible-light range, $t$ was calculated to be 0.36 , when $m=1$ and $n_{1}=2.08$ at $\lambda=550 \mathrm{~nm}$ were used. This value is consistent with the obtained slope of 0.39 , further confirming that the surface colors of these optical coatings could be precisely tuned by controlling $t$ of the AO thin films.

In the present study, the amorphous metals, semiconductors and insulators were derived from the same AA system. On the other hand, AA alloys usually show high hardness, excellent wear-resistance and corrosion- resistance [26-30]. In fact, the bilayer structured coatings showed a high hardness over $9 \mathrm{GPa}$, which may be used as the protective coatings with attractive decorative colors. High hardness means good wear resistant. The present colors belong to the category of structural colors. With the good mechanical properties, the color coatings are supposed to be stable, whose colors are difficult to fade with time. In addition, these color coatings usually work at room temperature. Even taking into account the heating effects in electronic devices, the working temperatures are normally below $500 \mathrm{~K}$. To evaluate the thermal stability of these colored coatings, we annealed the as-deposited golden and dark-grey thin films at $500 \mathrm{~K}$ for $2 \mathrm{~h}$. The reflectivity spectra of these annealed samples almost completely overlapped with those measured for the as-deposited bilayer thin films, respectively (Fig. S1). These results indicate that the colored coatings could work with high stability when used at temperatures below $500 \mathrm{~K}$ in the practical application.

\section{CONCLUSIONS}

In this study, adding oxygen into an amorphous metal was proven to be a simple and effective way for developing new amorphous materials ranging from metal to semiconductor, and eventually to insulator. These amorphous derivatives showed continuously tunable optical properties. Integrating reflective AA and transparent AO thin films enabled the development of high-resolution color coatings.

\section{Received 23 September 2020; accepted 1 February 2021;} published online 15 April 2021

1 Stillinger FH. A topographic view of supercooled liquids and glass formation. Science, 1995, 267: 1935-1939

2 Huang PY, Kurasch S, Alden JS, et al. Imaging atomic rearrangements in two-dimensional silica glass: Watching silica's dance. Science, 2013, 342: 224-227 
3 Luo P, Wen P, Bai HY, et al. Relaxation decoupling in metallic glasses at low temperatures. Phys Rev Lett, 2017, 118: 225901

4 Zhu F, Nguyen HK, Song SX, et al. Intrinsic correlation between $\beta$ relaxation and spatial heterogeneity in a metallic glass. Nat Commun, 2016, 7: 11516

5 Yamane M, Asahara Y, Asahara Y. Glasses for Photonics. London: Cambridge University Press, 2000

6 Ren J, Li K, Yang J, et al. Solution-processed amorphous galliumtin oxide thin film for low-voltage, high-performance transistors. Sci China Mater, 2019, 62: 803-812

7 Jiang ZH, Zhang QY. The formation of glass: a quantitative perspective. Sci China Mater, 2015, 58: 378-425

8 Nai J, Kang J, Guo L. Tailoring the shape of amorphous nanomaterials: recent developments and applications. Sci China Mater, 2015, 58: 44-59

9 Hosseini P, Wright CD, Bhaskaran H. An optoelectronic framework enabled by low-dimensional phase-change films. Nature, 2014, 511: 206-211

10 Yang C, Zhang C, Liu L. Tuning colors in Zr-based thin film metallic glasses. J Alloys Compd, 2017, 728: 289-294

$11 \mathrm{Bu} \mathrm{Y,} \mathrm{Bu} \mathrm{XM,} \mathrm{Lyu} \mathrm{FC,} \mathrm{et} \mathrm{al.} \mathrm{Full-color} \mathrm{reflective} \mathrm{filters} \mathrm{in} \mathrm{a} \mathrm{large}$ area with a wide-band tunable absorber deposited by one-step magnetron sputtering. Adv Opt Mater, 2019, 8: 1901626

12 Harmon JS, Demetriou MD, Johnson WL, et al. Anelastic to plastic transition in metallic glass-forming liquids. Phys Rev Lett, 2007, 99: 135502

13 Cheng YQ, Ma E. Atomic-level structure and structure-property relationship in metallic glasses. Prog Mater Sci, 2011, 56: 379-473

14 Ding J, Cheng YQ, Sheng H, et al. Universal structural parameter to quantitatively predict metallic glass properties. Nat Commun, 2016, 7: 13733

15 Wang WH. The elastic properties, elastic models and elastic perspectives of metallic glasses. Prog Mater Sci, 2012, 57: 487-656

16 Llordés A, Garcia G, Gazquez J, et al. Tunable near-infrared and visible-light transmittance in nanocrystal-in-glass composites. Nature, 2013, 500: 323-326

17 Liu W, Zhang H, Shi JA, et al. A room-temperature magnetic semiconductor from a ferromagnetic metallic glass. Nat Commun, 2016, 7: 13497

18 Chen $\mathrm{N}$, Fang $\mathrm{K}$, Zhang $\mathrm{H}$, et al. Amorphous magnetic semiconductors with Curie temperatures above room temperature. J Semicond, 2019, 40: 081510

19 Thomas G. Invisible circuits. Nature, 1997, 389: 907-908

20 Wager JF. Applied physics: transparent electronics. Science, 2003, 300: $1245-1246$

21 Nomura K, Ohta $\mathrm{H}$, Takagi A, et al. Room-temperature fabrication of transparent flexible thin-film transistors using amorphous oxide semiconductors. Nature, 2004, 432: 488-492

22 Wager JF, Keszler DA, Presley RE. Transparent electronics. New York: Springer Science+Business Media, LLC, 2008

23 Kamiya T, Hosono H. Material characteristics and applications of transparent amorphous oxide semiconductors. NPG Asia Mater, 2010, 2: 15-22

24 Kats MA, Blanchard R, Genevet P, et al. Nanometre optical coatings based on strong interference effects in highly absorbing media. Nat Mater, 2013, 12: 20-24

25 Macleod MC. Thin-film Optical Filters (4th edition). Boca Raton: CRC Press/Taylor \& Francis, 2010

26 Inoue A. Stabilization of metallic supercooled liquid and bulk amorphous alloys. Acta Mater, 2000, 48: 279-306
27 Johnson WL. Bulk glass-forming metallic alloys: science and technology. MRS Bull, 1999, 24: 42-56

28 Greer AL. Metallic glasses. Science, 1995, 267: 1947-1953

29 Wang WH, Dong C, Shek CH. Bulk metallic glasses. Mater Sci Eng-R-Rep, 2004, 44: 45-89

30 Chu JP, Huang JC, Jang JSC, et al. Thin film metallic glasses: Preparations, properties, and applications. J Miner Metals Mater Soc, 2010, 62: 19-24

Acknowledgements This work was financially supported by the National Science Fund for Excellent Young Scholars (51922053), and the School of Materials Science and Engineering at Tsinghua University. Zhong XY is grateful for the funding from the National Key Research and Development Program (2016YFB0700402), the National Natural Science Foundation of China (51822105 and 11834009), the City University of Hong Kong (9610484) and the Shenzhen Research Institute, the City University of Hong Kong. This work made use of the resources of the National Center for Electron Microscopy in Beijing. We thank Mr. Guo ZL for technique support.

Author contributions Zhang YQ, Zhou LY, Tao SY, Jiao YZ, Zheng $\mathrm{KM}$ and Fang KX contributed to the experimental tests. Xu L and Chen $\mathrm{N}$ co-wrote the manuscript and led the direction of the work. Chen $\mathrm{N}$ conceived the project and supervised the entire research. All authors contributed to the discussion, interpretation and presentation of the results.

Conflict of interest The authors declare that they have no conflict of interest.

Supplementary information Supporting data are available in the online version of the paper.

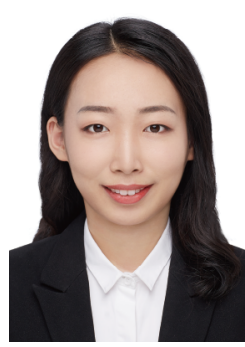

Yingqi Zhang received her Master's degree in materials science and engineering from Tsinghua University. During her study at Tsinghua University, she focused her research on the development and properties of new magnetic semiconductors.

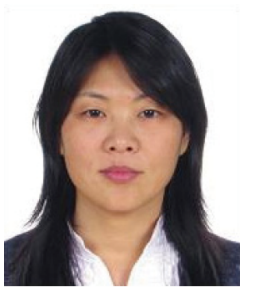

Limei Xu received her $\mathrm{PhD}$ degree in physics from Boston University in 2007. From 2008 to 2010, she was an assistant professor at the World Premier International Research Center InitiativeAdvanced Institute for Materials Research (WPIAIMR), Tohoku University of Japan. Then she became an associate professor at the International Center of Quantum Materials (ICQM), Peking University. Now she is a full professor of ICQM, Peking University. Her current research interests are mainly focused on soft condensed matter physics and computational physics. 


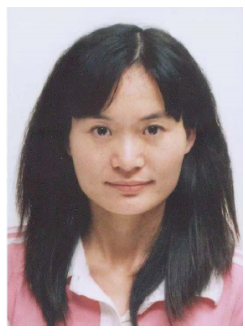

$\mathrm{Na}$ Chen received her $\mathrm{PhD}$ degree in materials science and engineering from Tsinghua University in 2008. She was a research associate at the WPI-AIMR, Tohoku University of Japan in 2008, where she became an assistant professor in 2010. Now, she is an associate professor at the School of Materials Science and Engineering, Tsinghua University. Her current research interests are focused on the design and development of new types of non-equilibrium nanomaterials and their potential applications.

\section{基于非晶合金体系的氧调控实现宽范围光学性能} 的精准可调

张盈祺 ${ }^{1}$, 周丽颖 ${ }^{2,3}$, 陶圣叶 ${ }^{1}$, 焦宇漳 ${ }^{1}$, 李晋锋 ${ }^{4}$, 郑凯鸣 ${ }^{1}$, 胡远超 ${ }^{5}$, 方凯旋, 宋成 ${ }^{6}$, 钟㙈龑 ${ }^{7,8}$, 徐莉梅 ${ }^{2,3^{*}}$, 姚可夫 ${ }^{1}$, 张政军 ${ }^{6}$, 陈娜 $^{1 *}$

摘要 宽范围精准调控非晶材料的光学性能是面向光电器件等潜 在应用亟需解决的关键问题之一. 研究发现, 通过在非晶合金CoFe-Ta-B体系中引入氧可以诱导金属-绝缘体转变, 从而制备出一系 列涵盖金属、半导体和绝缘体的非晶态衍生物. 随着氧含量的不 断增加, 这些含氧非晶态衍生物的光学带隙打开, 薄膜逐渐变得透 明. 复合高反射率的非晶合金和高透过率的非晶氧化物形成的双 层薄膜结构可以实现可见光波段的全色谱可调. 该研究结果为研 制具有高硬度、高耐磨性、设计制备简便、全色谱可调等优异性 能的大面积彩色涂层提供了一条新的途径. 同时, 该研究提出的设 计理念可能适用于已报道的很多非晶合金体系，通过这种氧调控 方式可制备出涵盖金属、半导体和绝缘体所有导电类型的非晶材 料, 用于研制基于这些材料光学功能特性的器件. 